\title{
Improving Intrinsic Decoherence in Multiple-Quantum-Dot Charge Qubits
}

\author{
Martina Hentschel, ${ }^{1}$ Diego C. B. Valente, ${ }^{2}$ Eduardo R. Mucciolo, ${ }^{2}$ and Harold U. Baranger ${ }^{3}$ \\ ${ }^{1}$ Max-Planck-Institut für Physik Komplexer Systeme, Nöthnitzer Straße 38, Dresden, Germany \\ ${ }^{2}$ Department of Physics, University of Central Florida, \\ P.O. Box 162385, Orlando, Florida 32816-2385, USA, and \\ ${ }^{3}$ Department of Physics, Duke University, P.O. Box 90305, Durham, North Carolina 27708-0305, USA
}

(Dated: November 30, 2018; arXiv:0705.3923)

\begin{abstract}
We discuss decoherence in charge qubits formed by multiple lateral quantum dots in the framework of the spin-boson model and the Born-Markov approximation. We consider the intrinsic decoherence caused by the coupling to bulk phonon modes. Two distinct quantum dot configurations are studied: (i) Three quantum dots in a ring geometry with one excess electron in total and (ii) arrays of quantum dots where the computational basis states form multipole charge configurations. For the three-dot qubit, we demonstrate the possibility of performing one- and two-qubit operations by solely tuning gate voltages. Compared to a previous proposal involving a linear three-dot spin qubit, the three-dot charge qubit allows for less overhead on two-qubit operations. For small interdot tunnel amplitudes, the three-dot qubits have $Q$ factors much higher than those obtained for double-dot systems. The high-multipole dot configurations also show a substantial decrease in decoherence at low operation frequencies when compared to the double-dot qubit.
\end{abstract}

PACS numbers: 73.21.La, 03.67.Lx, 73.23.Hk

\section{INTRODUCTION}

The realization of a solid-state qubit based on familiar and highly developed semiconductor technology would facilitate scaling to a many-qubit computer and make quantum computation more accessible. $\frac{1}{-1}$ The earliest proposal of a quantum dot qubit relied on the manipulation of the spin degree of freedom of a single confined electron..$^{2}$ An attractive point of that proposal is the large spin decoherence time characteristic of semiconductors; a drawback is that it requires local control of intense magnetic fields. As an alternative, a spin-based logical qubit involving a multiple quantum dot setup and voltagecontrolled exchange interactions was devised, $\frac{3}{-}$ but at the price of considerable overhead in additional operations.

While spin qubits remain promising in the long term - note in particular several recent experimental advances ${ }^{4,5}$ as well as further theoretical development of multiple-quantum dot spin qubits ${ }^{6}-$ charge-based qubits in quantum dots, in analogy to superconducting Cooper-pair box devices, $, 8,8,9,10$ are also worthy of investigation. Employing the charge degree of freedom of electrons rather than their spin brings a few important practical advantages: No local control of magnetic fields is required and all operations can be carried out by manipulating gate voltages. The simplest realization of a charge qubit is a double quantum dot system with an odd number of electrons. ${ }^{11,12,13,14,15,16,17}$ One can view this system as a double well potential: The unpaired electron moves between the two wells (i.e., quantum dots) by tunneling through the potential barrier. The logical states $|0\rangle,|1\rangle$ correspond to the electron being on the left or right. The barrier height determines the tunneling rate between the dots and can be adjusted by a gate voltage. The resulting bonding and antibonding states can also be used as the computational basis. Recently, three groups have implemented the double-dot charge qubit experimentally $18,19,20$

Charge qubits are susceptible to various decoherence mechanisms related to charge motion. Strong damping of coher- ent oscillations was observed in all quantum dot experimental setups, $\frac{18,19,20}{2}$ with quality factors in the range $3-10$. Note that a change in the state of the qubit involves electron motion between quantum dots, which can in general couple very effectively to external degrees of freedom such as phonons, charge traps in the substrate, and electromagnetic environmental fluctuations. These noise sources lead to decoherence times much shorter than those observed in spin qubit systems. Thus, one is tempted to try to find new setups where oscillations between qubit states involve a minimum amount of charge motion. For instance, in qubits based on multiple quantum dots one can pick logical states where charge is homogeneously distributed in space. Another approach is to create a multiple dot structure with symmetries that forbid coupling to certain environmental modes within the logical subspace ${ }^{21}$ A recent attempt along this direction is found in Ref. 22.

In this paper we argue that it is not generally possible to avoid decoherence in multiple-quantum-dot charge qubits by simple geometrical constructions. The spreading of charge uniformly over a multiple-quantum-dot logical qubit does not avoid decoherence. However, the coupling to bosonic environmental modes, such as phonons and photons, can be very substantially attenuated in some circumstances.

In order to demonstrate these assertions, we analyze in detail two prototypical extensions of the double-quantum dot charge qubit. We first consider a qubit consisting of three quantum dots forming a ringlike structure and only one extra electron, as shown in Fig. 1. Multiple-quantum-dot qubits with a ringlike structure resemble a proposal by Kulik et al ${ }^{23}$ to use persistent current states in metallic rings for quantum computation. Unlike the double-dot qubit case, the ground state in a three-dot qubit can be truly degenerate with corresponding wave functions having a uniform charge distribution. At first, this raises the hope that decoherence mechanisms involving charge inhomogeneities (such as phonons or charge traps) would be inhibited due to mutual cancellations. However, we shall see below that the computational 


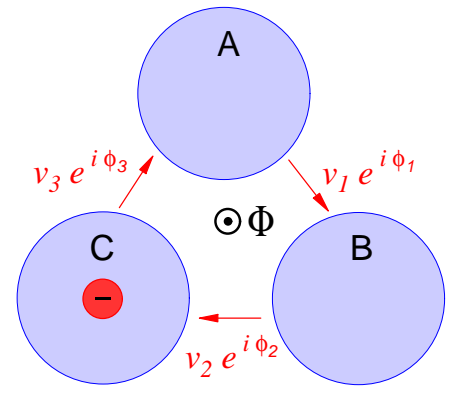

FIG. 1: (Color online) Schematic illustration of a three-quantum-dot qubit with only one extra, unpaired electron. The external tuning parameters are the strength of the tunneling couplings $\left(v_{1}, v_{2}\right.$, and $v_{3}$ ) and the magnetic flux $\Phi=\phi_{1}+\phi_{2}+\phi_{3}$ through the qubit. The latter is used solely to define the working point of the qubit.

basis states can be distinguished by phonon and electromagnetic baths through the electron phase variations along the ring. That, in turn, leads to dephasing and decoherence. This problem is intrinsic to all quantum-dot-based charge qubits. Nevertheless, the $Q$ factor in these three dot qubits can be 1-2 orders of magnitude larger than in the corresponding doubledot qubits, a substantial improvement in coherence.

Second, we show that planar quantum dot arrays in the form of high-order multipoles can be more efficient in reducing the coupling to acoustic phonons in multiple-quantum dot qubits. Our work extends and analyzes in detail a recent proposal to create a decoherence-free subspace with charge qubits ${ }^{22}$

While it is well known that condensed-matter environments tend to produce time and spatial correlations in their interaction with qubits, 24 here we assume that the Markov approximation provides reasonable estimates of decoherence rates. In particular, we employ the Redfield equations in the weakcoupling, Born-Markov approximation to describe the time evolution of the reduced density matrix of the qubit system. ${ }^{25}$

The paper is organized as follows. In Sec. IIwe study in detail a three-dot charge qubit. Single- and two-qubit operations are presented, as well as the coupling to a bosonic bath. We consider in detail the particular case of acoustic piezoelectric phonons, which is relevant to III-V semiconductor materials at low temperatures. Also in Sec. [I] we evaluate decoherence and energy relaxation rates using the Redfield equation formalism. In Sec. III we present a multiple-quantum dot logical qubit structure that minimizes the coupling to environmental modes which couple to charge. We also analyze phonon decoherence in these systems and compare $Q$ factors with those obtained with double-dot charge qubits. Finally, our conclusions are presented in Sec. IV The Appendix contains mathematical details of the two-qubit operation of the three-dot charge qubit of Sec. [II Throughout this paper we assume $\hbar=1$ and $k_{B}=1$.

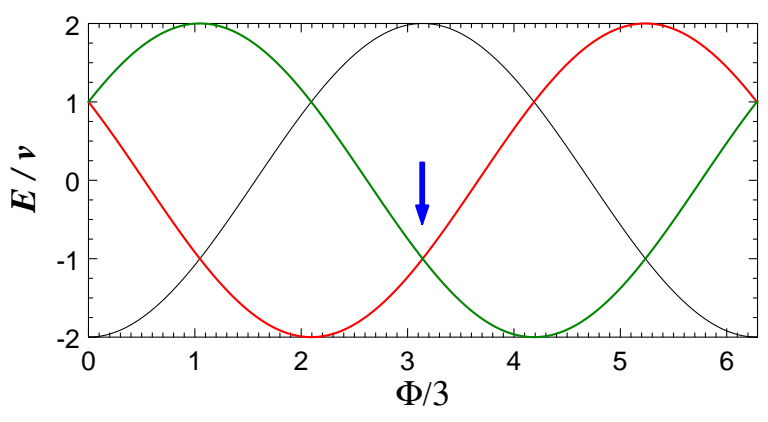

FIG. 2: (Color online) Eigenenergies of the three-dot qubit as function of the magnetic flux. The working point at $\Phi / 3=\phi=\pi$ per bond is indicated by the arrow. At this point, clockwise and counterclockwise persistent current states are degenerate, and the charge distribution is homogeneous throughout the space spanned by the computational basis.

\section{THE THREE-DOT CHARGE QUBIT}

A simple example of a multiple-dot qubit with charge delocalization consists of three quantum dots in a ringlike geometry, as shown in Fig. 11 In practice, this system is created by laterally confining electrons in a two-dimensional plane; the confinement is electrostatic, controlled through electrodes sitting above the plane. Consider gate voltages on the electrodes such that the three dots share one excess, unpaired electron, while all configurations with a different number of excess electrons become energetically inaccessible due to the large charging energy of the dots ${ }^{26}$ The spin degree of freedom is not relevant for our discussion and electrons will be assumed spinless unless otherwise specified. Thus, the system lives in a three-dimensional Hilbert space. The electron can hop between dots through tunneling. The tunneling matrix elements and the on-site energies are controlled by the gate voltages. As will be clear shortly, it is convenient to apply a weak magnetic field perpendicular to the plane containing the dots.

The three natural basis states place the electron on $\operatorname{dot} \mathrm{A}$, $\mathrm{B}$, or $\mathrm{C}$ :

$$
\left.\left.\left.|A\rangle=c_{A}^{\dagger} \mid \text { vac. }\right\rangle, \quad|B\rangle=c_{B}^{\dagger} \mid \text { vac. }\right\rangle, \quad|C\rangle=c_{C}^{\dagger} \mid \text { vac. }\right\rangle,
$$

where $c_{\alpha}^{\dagger}$ are creation operators and $\mid$ vac. $\rangle$ is a reference state where all dots have an even number of electrons. In this basis, the Hamiltonian takes the matrix form

$$
H=\left(\begin{array}{ccc}
E_{A} & -v_{1} e^{i \phi_{1}} & -v_{3} e^{-i \phi_{3}} \\
-v_{1} e^{-i \phi_{1}} & E_{B} & -v_{2} e^{i \phi_{2}} \\
-v_{3} e^{i \phi_{3}} & -v_{2} e^{-i \phi_{2}} & E_{C}
\end{array}\right),
$$

where $E_{A}, E_{B}$, and $E_{C}$ are the on-site energies, $v_{i}$ are the tunneling strengths between pairs of quantum dots, and $\phi_{1}+$ $\phi_{2}+\phi_{3}=\Phi$ is the total magnetic flux through the ring. Let us specify the qubit by setting $v_{1}=v_{2}=v_{3} \equiv v>0$, $E_{A}=E_{B}=E_{C} \equiv 0$, and $\phi_{1}=\phi_{2}=\phi_{3}=\Phi / 3 \equiv \pi$. In this configuration, two degenerate eigenstates $|+\rangle$ and $|-\rangle$ have the lowest energy, $E_{ \pm}=-v$ (Fig. 2). They carry clockwise 
and counterclockwise persistent currents and form the computational basis. ${ }^{27}$ The third, excited, eigenstate $|T\rangle$ has energy $E_{e}=2 v$ and is current-free. The eigenvectors are

$$
\begin{aligned}
& |T\rangle=\frac{1}{\sqrt{3}}(|A\rangle+|B\rangle+|C\rangle), \\
& |+\rangle=\frac{1}{\sqrt{3}}\left(|A\rangle+e^{i \beta}|B\rangle+e^{-i \beta}|C\rangle\right), \\
& |-\rangle=\frac{1}{\sqrt{3}}\left(|A\rangle+e^{-i \beta}|B\rangle+e^{i \beta}|C\rangle\right),
\end{aligned}
$$

with $\beta=2 \pi / 3$. Clearly, the charge distribution is spatially uniform for all three states.

It is worth noting that the topology of the three-dot qubit and its use of persistent currents of opposite direction as logical states closely resemble the Josephson persistent current qubit studied in Ref. 28 or the proposed atomic Josephson junction arrays ${ }^{29}$ However, the similarities stop here as the underlying physics is very different. We will focus our discussion on the quantum dot charge qubit case only.

\section{A. Single-qubit operations}

In order to be able to perform quantum gate operations, we have to allow for deviations from the degeneracy point. This is done by varying the tunneling coupling and/or the magnetic flux. It is convenient to introduce the (small) parameters $\delta_{1}$, $\delta_{2}, \delta_{3}$, and $\varphi$ such that $v_{1}=v+\delta_{1}, v_{2}=v+\delta_{2}, v_{3}=v+\delta_{3}$, and $\varphi \ll 1$ with $\varphi=\Phi-3 \pi$. To linear order and using a $\{|T\rangle,|+\rangle,|-\rangle\}$ basis, we find that the Hamiltonian expanded around the degeneracy point can be written as

$$
H=\left(\begin{array}{ccc}
2 v+\frac{2}{3}\left(\delta_{1}+\delta_{2}+\delta_{3}\right) & -\frac{1}{3}\left(\delta_{1} e^{-i \beta}+\delta_{2}+\delta_{3} e^{i \beta}\right) & -\frac{1}{3}\left(\delta_{1} e^{i \beta}+\delta_{2}+\delta_{3} e^{-i \beta}\right) \\
-\frac{1}{3}\left(\delta_{1} e^{i \beta}+\delta_{2}+\delta_{3} e^{-i \beta}\right) & -v-v \varphi / \sqrt{3}-\frac{1}{3}\left(\delta_{1}+\delta_{2}+\delta_{3}\right) & \frac{2}{3}\left(\delta_{1} e^{-i \beta}+\delta_{2}+\delta_{3} e^{i \beta}\right) \\
-\frac{1}{3}\left(\delta_{1} e^{-i \beta}+\delta_{2}+\delta_{3} e^{i \beta}\right) & \frac{2}{3}\left(\delta_{1} e^{i \beta}+\delta_{2}+\delta_{3} e^{-i \beta}\right) & -v+v \varphi / \sqrt{3}-\frac{1}{3}\left(\delta_{1}+\delta_{2}+\delta_{3}\right)
\end{array}\right)
$$

The computational subspace corresponds to the lower-right $2 \times 2$ block. Evidently, we stay within the computational subspace as long as $\delta_{1}=\delta_{2}=\delta_{3}$. However, this also implies that there is no coupling between the computational basis states $|+\rangle$ and $|-\rangle$. For $\delta_{1} e^{i \beta}+\delta_{2}+\delta_{3} e^{-i \beta} \neq 0$, coupling within the computational subspace is possible, but there is a finite probability of leaking out into the state $|T\rangle$. The leakage can be kept small as long as $v \gg\left|\delta_{1,2,3}\right|$. Alternatively, one can incorporate the third level into the single-qubit operations, as in Ref.23. For the following case study, we assume that the leakage from the computational subspace is negligible.

Using the Pauli matrices $\sigma_{1}, \sigma_{2}$, and $\sigma_{3}$, as well as the identity matrix $\sigma_{0}$, we can express the Hamiltonian in the computational basis in terms of a pseudospin in a pseudomagnetic field $\vec{h}$ plus a constant,

$$
H_{S}=E_{0} \sigma_{0}+h_{x} \sigma_{1}+h_{y} \sigma_{2}+h_{z} \sigma_{3},
$$

where $E_{0}=-v-\left(\delta_{1}+\delta_{2}+\delta_{3}\right) / 3$ and

$$
\begin{aligned}
h_{x} & =\frac{2}{3}\left(\delta_{2}-\frac{\delta_{1}+\delta_{3}}{2}\right), \\
h_{y} & =\frac{\delta_{1}-\delta_{3}}{\sqrt{3}}, \\
h_{z} & =-v \varphi / \sqrt{3} .
\end{aligned}
$$

We only need to vary two out of the three pseudomagnetic field components in order to perform single-qubit operations. Thus, we can operate the qubit at constant magnetic flux (and set $\left.\varphi=0, h_{z}=0\right)$ and vary only the $\delta_{i}$ via gate voltages. If we furthermore fix the coupling $v_{2} \equiv v, \delta_{2}=0$, we find that the qubit is controlled by the sum and difference of the

variation of two intra-qubit couplings, $h_{x} \propto\left(\delta_{1}+\delta_{3}\right)$ and $h_{y} \propto\left(\delta_{1}-\delta_{3}\right)$, that can be adjusted by tuning the respective gate voltages around the symmetry point.

\section{B. Two-qubit operations}

In order to perform two-qubit operations, such as the SWAP or CNOT gate, we have to couple two three-dot qubits (called I and II hereafter). In principle, this can be done in either a tip-to-tip or base-to-base coupling scheme, as shown in Fig. 3. Since the number of excess electrons in the composite system is equal to two, states where two electrons occupy the same qubit have to be included in the basis of the two-qubit Hilbert

(a)
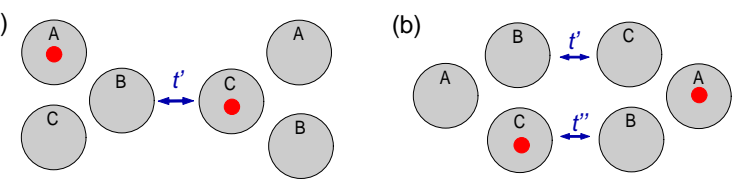

(c)

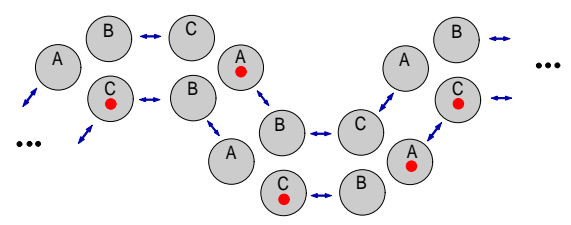

FIG. 3: (Color online) Possible implementations of two-qubit gates using three-dot qubits. (a) Coupling via a single dot (tip-tip geometry); (b) coupling via two dots (base-base geometry). (c) A possible implementation of a qubit chain in the base-base configuration. 
space. The basis of the two-qubit Hilbert space reads thus

$$
\begin{aligned}
|1\rangle & =|+\rangle_{\mathrm{I}}|+\rangle_{\mathrm{II}}, \\
|2\rangle & =|+\rangle_{\mathrm{I}}|-\rangle_{\mathrm{II}}, \\
|3\rangle & =|-\rangle_{\mathrm{I}}|+\rangle_{\mathrm{II}}, \\
|4\rangle & =|-\rangle_{\mathrm{I}}|-\rangle_{\mathrm{II}}, \\
|5\rangle & \left.=c_{A \mathrm{I}}^{\dagger} c_{B \mathrm{I}}^{\dagger} \mid \text { vac. }\right\rangle, \\
|6\rangle & \left.=c_{A \mathrm{I}}^{\dagger} c_{C \mathrm{I}}^{\dagger} \mid \text { vac. }\right\rangle, \\
|7\rangle & \left.=c_{B \mathrm{I}}^{\dagger} c_{C \mathrm{I}}^{\dagger} \mid \text { vac. }\right\rangle, \\
|8\rangle & \left.=c_{A \mathrm{II}}^{\dagger} c_{B \mathrm{II}}^{\dagger} \mid \text { vac. }\right\rangle, \\
|9\rangle & \left.=c_{A \mathrm{II}}^{\dagger} c_{C \mathrm{II}}^{\dagger} \mid \text { vac. }\right\rangle, \\
|10\rangle & \left.=c_{B \mathrm{II}}^{\dagger} c_{C \mathrm{II}}^{\dagger} \mid \text { vac. }\right\rangle .
\end{aligned}
$$

Here, two types of states have been neglected: First, states with double occupancy of a single dot since the charging energy is assumed to be very large. Second, although the $|T\rangle_{\mathrm{I}}$ and $|T\rangle_{\text {II }}$ states couple to the double-occupied states $|5\rangle$ to $|10\rangle$ through the inter-qubit hopping terms, they are gapped by an energy of order $v$, which is assumed much larger than the effective two-qubit interaction amplitude $t^{\prime 2} / U_{i}$ (see below). Therefore, they were not included in the two-qubit Hilbert subspace. 30

The Hamiltonian for the inter-qubit interaction in the tip-tip setting shown in Fig. 3 (a) reads

$$
H_{\mathrm{I}-\mathrm{II}}^{\mathrm{tip}}=-t^{\prime}\left(c_{B \mathrm{I}}^{\dagger} c_{C \mathrm{II}}+c_{C \mathrm{II}}^{\dagger} c_{B \mathrm{I}}\right) \text {. }
$$

Similarly, the base-base coupling presented in Fig.3 (b) is governed by the Hamiltonian (see also the Appendix)

$$
H_{\mathrm{I}-\mathrm{II}}^{\mathrm{base}}=-t^{\prime}\left(c_{B \mathrm{I}}^{\dagger} c_{C \mathrm{II}}+c_{C \mathrm{II}}^{\dagger} c_{B \mathrm{I}}\right)-t^{\prime \prime}\left(c_{C \mathrm{I}}^{\dagger} c_{B \mathrm{II}}+c_{B \mathrm{II}}^{\dagger} c_{C \mathrm{I}}\right),
$$

where we have chosen the gauge for the vector potential associate to the perperdincular magnetic field to be parallel to the inter-qubit tunneling paths. We assume that the inter-qubit tunneling amplitudes $t^{\prime}$ and $t^{\prime \prime}$ satisfy $0<t^{\prime}, t^{\prime \prime} \ll v \ll U_{i}$, where $U_{i}$ is the inter-dot charging or capacitive coupling energy (i.e., the change in the energy of one dot when an electron is added to one of the neighboring dots). In other words, the capacitive coupling between dots must be sufficiently strong so that states with two or zero excess electrons in a qubit are forbidden. Due to the proximity between dots of neighboring qubits, some small inter-qubit capacitive coupling will also exist. Although we will neglect such coupling in the discussion below, these additional charging energies can be included without substantially modifying our results. In particular, we note that the inter-qubit capacitive coupling does not interfere with single-qubit operations. Note also that the presence of a magnetic flux requires the dots $\mathrm{A}, \mathrm{B}$, and $\mathrm{C}$ to be always arranged in a clockwise order.

Next, the large charging energy separation between the single-occupancy states $|1\rangle$ to $|4\rangle$ and the double-occupancy states $|5\rangle$ to $|10\rangle$ allows us to separate the two-qubit computational subspace from the rest of the Hilbert space. In order to do so, we use a Schrieffer-Wolff transformation, ${ }^{31}$ which amounts to a second-order perturbative expansion of the effective Hamiltonian in the ratio of the inter-qubit tunneling magnitude to the charging energy. To this end we insert the expressions for $|+\rangle$ and $|-\rangle$ from Eqs. (4)-(5) into Eqs. (11)-(14) and express the computational basis states $|1\rangle$ to $|4\rangle$ in terms of creation operators acting on the vacuum state. Further, using the basis vectors in Eqs. (11) to (20), one can easily compute the full six-dot Hamiltonian in the basis of states $|1\rangle$ to $|10\rangle$. Noting that one can obtain the tip-tip Hamiltonian from the expression for the base-base case by setting $t^{\prime \prime}=0$, we evaluate the more general case of the base-base coupling, see Fig. 3b and Eq. (22). The details of the computation, i.e. the full matrix representation of this Hamiltonian, as well as its reduction to the two-qubit computational basis by performing the Schrieffer-Wolff transformation, are shown in Appendix A. The result for the reduced Hamiltonian takes a rather compact form which, for the tip-tip case, reads

$$
\tilde{H}_{\mathrm{I}-\mathrm{II}}^{\mathrm{tip}}=-\frac{t^{\prime 2}}{9 U_{i}}\left(\begin{array}{cccc}
4 & e^{-i \beta} & e^{i \beta} & -2 \\
e^{i \beta} & 4 & -2 e^{-i \beta} & e^{i \beta} \\
e^{-i \beta} & -2 e^{i \beta} & 4 & e^{-i \beta} \\
-2 & e^{-i \beta} & e^{i \beta} & 4
\end{array}\right) .
$$

Note that this reduced Hamiltonian acts on the subspace formed by the states $\{|1\rangle, \ldots|4\rangle\}$ defined in Eqs. (11) to (14). $\mathrm{Up}$ to the common prefactor $-t^{\prime 2} /\left(9 U_{i}\right)$, the eigenvalues of $\tilde{H}_{\mathrm{I}-\mathrm{II}}^{\text {tip }}$ are $E_{1}=0, E_{2}=4, E_{3}=6$, and $E_{4}=6$, with the respective eigenvectors equal to

$$
\begin{aligned}
& \left|E_{1}\right\rangle=\frac{1}{2}\left(|1\rangle-e^{i \beta}|2\rangle-e^{-i \beta}|3\rangle+|4\rangle\right), \\
& \left|E_{2}\right\rangle=\frac{1}{2}\left(|1\rangle+e^{i \beta}|2\rangle+e^{-i \beta}|3\rangle+|4\rangle\right), \\
& \left|E_{3}\right\rangle=\frac{1}{\sqrt{2}}(|1\rangle-|4\rangle), \\
& \left|E_{4}\right\rangle=\frac{1}{\sqrt{2}}\left(e^{i \beta}|2\rangle-e^{-i \beta}|3\rangle\right) .
\end{aligned}
$$

The critical question now is whether this setup permits a convenient two-qubit operation, such as a full SWAP. It is straightforward to show that the answer is positive, even in the simple tip-tip coupling scheme. To see that, suppose we initialize the qubits in state $|2\rangle$ and now search for the time $\tau$ after which the qubits have evolved onto the (swapped) state $|3\rangle$ under the action of $\tilde{H}_{\mathrm{I}-\mathrm{II}}^{\mathrm{tip}}$. The square of the resulting condition, $\left|\left\langle 3\left|e^{-i \tilde{H}_{\mathrm{I}-\mathrm{II}}^{\mathrm{tip}} \tau}\right| 2\right\rangle\right|^{2} \equiv 1$, is readily evaluated and yields $\tau_{S}=\pi / 2\left[t^{\prime 2} /\left(9 U_{i}\right)\right]^{-1}$ as the (shortest) time for which the tip-tip coupling $t^{\prime}$ has to be turned on in order to implement the SWAP gate.

For a comparison with the (linear) three-dot spin qubit scheme proposed by DiVincenzo et al., $\frac{3}{\underline{3}}$ let us briefly discuss the implementation of the CNOT quantum gate. A CNOT can be done straightforwardly using two $\sqrt{\text { SWAP }}$ operations (SWAP gates of duration $\tau_{S} / 2$ ) and seven one-qubit gates, 2,32 e.g., by utilizing the scheme in Ref.32. Consequently, we find that the realization of one- and two-qubit operations for the present three-dot charge qubit is considerably simpler than for the proposal by DiVincenzo et al. where many more steps 
were necessary to implement a CNOT. One reason is the complexity of the one-qubit rotations - for the logical spin-qubit, one-qubit operations alone require three spin exchange interaction pulses. For the CNOT gate, this implies at least 19 pulses with 11 different operation times. Compared to the 9 pulses needed for the three-dot charge qubit, the practical advantages of the qubit and computation scheme proposed here are evident.

\section{Coupling to a bosonic bath}

The charge qubit couples to a variety of environmental degrees of freedom. We study in particular the decoherence caused by gapless bosonic modes that sense charge fluctuations in the dots, such as phonons. We assume that all quantum dots couple to the same bath. The Hamiltonian describing the non-interacting bosonic modes in this case is

$$
H_{B}=\sum_{\mathbf{q}} \omega_{\mathbf{q}} b_{\mathbf{q}}^{\dagger} b_{\mathbf{q}}
$$

with q denoting the boson linear momentum and $\omega_{\mathbf{q}}$ its dispersion relation. The coupling between the dots and the bosons is assumed to be governed by the bilinear Hamiltonian ${ }^{33}$

$$
H_{\mathrm{dot}-\text { boson }}=\sum_{\mathbf{q}}\left(\alpha_{A} N_{A}+\alpha_{B} N_{B}+\alpha_{C} N_{C}\right)\left(b_{\mathbf{q}}^{\dagger}+b_{-\mathbf{q}}\right)
$$

where $N_{k}$ is the number operator of the $k$ th dot, and

$$
\alpha_{k}=\lambda_{\mathbf{q}} P_{\mathbf{q}}^{(k)} e^{i \mathbf{R}_{k} \cdot \mathbf{q}}
$$

Here, $\lambda_{\mathbf{q}}$ represents the electron-boson coupling constant and $P_{\mathbf{q}}^{(k)}$ and $\mathbf{R}_{k}$ are form factor and position vector of the $k$ th dot, respectively. Note that all geometrical information is contained in the coefficients $\alpha_{k}$. Since we have exactly one excess electron on the three-dot system, the constraint $N_{A}+N_{B}+N_{C}=1$ must be satisfied. Therefore, the systembath Hamiltonian in the basis $\{|A\rangle,|B\rangle,|C\rangle\}$ reads

$$
H_{\mathrm{SB}}=\sum_{\mathbf{q}}\left(\begin{array}{ccc}
\alpha_{A} & 0 & 0 \\
0 & \alpha_{B} & 0 \\
0 & 0 & \alpha_{C}
\end{array}\right)\left(b_{\mathbf{q}}^{\dagger}+b_{-\mathbf{q}}\right)
$$

Projection of this Hamiltonian onto the subspace spanned by $|+\rangle$ and $|-\rangle$ defined in Eqs. (4) and (5) constrains the coupling to that subspace, yielding

$$
\tilde{H}_{\mathrm{SB}}=\frac{1}{3} \sum_{\mathbf{q}}\left[\left(\alpha_{A}-\frac{\alpha_{B}+\alpha_{C}}{2}\right) \sigma_{1}-\frac{\sqrt{3}}{2}\left(\alpha_{B}-\alpha_{C}\right) \sigma_{2}\right]\left(b_{\mathbf{q}}^{\dagger}+b_{-\mathbf{q}}\right),
$$

where a term proportional to $\sigma_{0}$ has been dropped. The presence of two terms with different functional dependence on $\mathbf{q}$ indicates the coupling to two bath modes, which will be denoted by the indices 1 and 2 in the following. There would be a third bath mode, proportional to $\sigma_{3}$, if the charge distribution were not the same for the two logical states. The advantage of having a homogeneous charge distribution for both states in the computational basis, leading directly to the cancellation of this third mode of decoherence, is evident here. It is important to remark that charge homogeneity can be achieved without the assumptions of homogeneous tunneling or equal capacitances: as long as one can tune the gate voltages in the quantum dots independently, one can arrange to have one extra electron equally shared among the three dots.

It is convenient to rewrite the system-bath Hamiltonian in the standard spin-boson form ${ }^{34}$

$$
\tilde{H}_{\mathrm{SB}} \equiv K_{1} \Phi_{1}+K_{2} \Phi_{2}
$$

where

$$
K_{1} \equiv \sigma_{1} / 6 \quad \text { and } \quad K_{2} \equiv-\sigma_{2} / 2 \sqrt{3}
$$

describe the system part and the corresponding bath part is given by $\Phi_{1,2}=\sum_{q} g_{\mathbf{q}}^{(1,2)}\left(b_{\mathbf{q}}^{\dagger}+b_{-\mathbf{q}}\right)$, with

$$
\begin{aligned}
& g_{\mathbf{q}}^{(1)}=2 \alpha_{A}-\alpha_{B}-\alpha_{C}, \\
& g_{\mathbf{q}}^{(2)}=\alpha_{B}-\alpha_{C} .
\end{aligned}
$$

Assuming all $P_{\mathbf{q}}^{(k)}$ to be the same, the following relations among the $\alpha_{k}$ can be obtained:

$$
\begin{aligned}
\alpha_{A} & =\lambda_{\mathbf{q}} P_{\mathbf{q}}, \\
\alpha_{B} & =\alpha_{A} e^{i\left(\mathbf{R}_{B}-\mathbf{R}_{A}\right) \cdot \mathbf{q}} \equiv \alpha_{A} e^{i \eta_{B}}, \\
\alpha_{C} & =\alpha_{A} e^{i\left(\mathbf{R}_{C}-\mathbf{R}_{A}\right) \cdot \mathbf{q}} \equiv \alpha_{A} e^{i \eta_{C}}
\end{aligned}
$$

where the last two equations define the phases $\eta_{B}$ and $\eta_{C}$. This completes the specification of the qubit-bath coupling.

\section{The Redfield equation}

We now investigate the qubit decoherence due to the bosonic bath by determining the time relaxation of the system's reduced density matrix. We use the Born and Markov approximations and the Redfield equation. 25 In this formalism the reduced density matrix of the system (qubit) is obtained by 
integrating out the bath degrees of freedom and assuming that: (i) the coupling to the bath is weak, so leading order perturbation theory is applicable (the Born approximation), and (ii) the bath correlation time is much shorter than the typical time scale of operation of the qubit, so that system-bath interaction events are uncorrelated in time (the Markov approximation).

The time evolution of the reduced density matrix is given by the Redfield equation 25,35

$$
\begin{aligned}
\dot{\rho}(t)= & -i\left[\tilde{H}_{S}(t), \rho(t)\right] \\
& +\sum_{\alpha=1,2}\left\{\left[\Lambda_{\alpha}(t) \rho(t), K_{\alpha}\right]+\left[K_{\alpha}, \Lambda_{\alpha}^{\dagger}(t) \rho(t)\right]\right\}
\end{aligned}
$$

where the time-dependent auxiliary matrices $\Lambda_{\alpha}(t)$ which encode the bath correlation properties are defined by

$$
\Lambda_{\alpha}(t)=\sum_{\beta=1,2} \int_{0}^{\infty} d t^{\prime} B_{\alpha \beta}\left(t^{\prime}\right) e^{-i t^{\prime} \tilde{H}_{S}(t)} K_{\beta} e^{i t^{\prime} \tilde{H}_{S}(t)}
$$

The thermal-average bath correlation functions,

$$
B_{\alpha \beta}(t)=\left\langle\Phi_{\beta}(t) \Phi_{\alpha}(0)\right\rangle
$$

can be written in terms of spectral functions,

$$
\nu_{\alpha \beta}(\omega)=\sum_{\mathbf{q}} g_{\mathbf{q}}^{(\alpha)} g_{-\mathbf{q}}^{(\beta)} \delta\left(\omega-\omega_{\mathbf{q}}\right)
$$

and the boson occupation number $n_{B}(\omega)=\left(e^{\omega / T}-1\right)^{-1}$ :

$$
\begin{aligned}
B_{\alpha \beta}(t)= & \int_{0}^{\infty} d \omega \nu_{\alpha \beta}(\omega) \\
& \left.\times\left\{e^{i \omega t} n_{B}(\omega)+e^{-i \omega t}\left[1+n_{B}(\omega)\right]\right)\right\} .
\end{aligned}
$$

Performing the sum over $\mathbf{q}$ in Eq. (43), we find

$$
\begin{aligned}
\nu_{11}= & 2 \sum_{\mathbf{q}}\left|\lambda_{\mathbf{q}} P_{\mathbf{q}}\right|^{2} \delta\left(\omega-\omega_{\mathbf{q}}\right) \\
& \times\left[3-2\left(\cos \eta_{B}-\cos \eta_{C}\right)+\cos \left(\eta_{B}-\eta_{C}\right)\right] \\
\nu_{22}= & 2 \sum_{\mathbf{q}}\left|\lambda_{\mathbf{q}} P_{\mathbf{q}}\right|^{2} \delta\left(\omega-\omega_{\mathbf{q}}\right)\left[1-\cos \left(\eta_{B}-\eta_{C}\right)\right] \\
\nu_{12}= & 2 \sum_{\mathbf{q}}\left|\lambda_{\mathbf{q}} P_{\mathbf{q}}\right|^{2} \delta\left(\omega-\omega_{\mathbf{q}}\right) \\
& \times\left[e^{-i \eta_{B}}-e^{-i \eta_{C}}+i \sin \left(\eta_{B}-\eta_{C}\right)\right]
\end{aligned}
$$

with $\nu_{21}=\nu_{12}^{*}$. When the bath is sufficiently large, the sums over the vector $q$ in Eqs. (45)-(47) can be converted into threedimensional integrals.

A few simplifying but realistic assumptions can be made at this point. Let us first assume that the coupling constant $\lambda_{\mathbf{q}}$ and the dispersion relation $\omega_{\mathbf{q}}$ are both isotropic. Second, let us assume that the electronic density in the dots has a Gaussian profile, $\rho(\mathbf{r})=\delta(z) e^{-r^{2} /\left(2 a^{2}\right)} /\left(2 \pi a^{2}\right)$, resulting in

$$
P_{\mathbf{q}}=\int d^{3} r \rho(r) e^{-i \mathbf{q} \cdot \mathbf{r}}=e^{-(a q \sin \theta)^{2} / 2},
$$

where $(q, \theta, \varphi)$ are the spherical coordinates of the boson wave vector. Then, the three-fold symmetry in the plane causes $\nu_{12}(\omega)$ to vanish and

$$
\nu_{11}(\omega)=3 \nu(\omega), \quad \nu_{22}(\omega)=\nu(\omega)
$$

with

$$
\begin{aligned}
\nu(\omega)= & \frac{\Omega q^{2}}{2 \pi^{2}}\left|\lambda_{q}\right|^{2}\left|\frac{d \omega_{q}}{d q}\right|^{-1} \int_{0}^{\pi / 2} d \theta \sin \theta e^{-(q a \sin \theta)^{2}} \\
& \times\left[1-J_{0}(q D \sin \theta)\right]
\end{aligned}
$$

where $\omega=\omega_{q}, \Omega$ is the crystal unit cell volume, and $D$ is the distance between dots.

For III-V semiconductor materials at low temperatures, the most relevant bosonic modes are piezoelectric acoustic phonons, ${ }^{37}$ for which we have $\lambda_{q}=\pi s \sqrt{g_{\mathrm{ph}} / q \Omega}$ and $\omega_{q}=$ $s q$. Here, $g_{\mathrm{ph}}$ is the dimensionless electron-phonon coupling constant and $s$ is the phonon velocity (for GaAs, $g_{\mathrm{ph}} \approx 0.05$ and $\left.s \approx 5 \times 10^{3} \mathrm{~m} / \mathrm{s}\right) .15$

\section{E. Decoherence rates}

We now solve the equation-of-motion for the reduced density matrix explicitly for a case in which the decoherence rate can be obtained directly. Consider a constant pulse applied to the qubit at $t=0$ such that $h_{y}=h_{z}=0$ and $h_{x}=\Delta>0$. For $t>0$, the $\Lambda_{\alpha}$ matrices are constant and given by

$$
\begin{aligned}
& \Lambda_{1}=\gamma_{0} \sigma_{1} / 2 \\
& \Lambda_{2}=-(1 / 2 \sqrt{3})\left(\gamma_{c} \sigma_{2}+\gamma_{s} \sigma_{3}\right)
\end{aligned}
$$

The (complex) relaxation rates are given by

$$
\begin{aligned}
\gamma_{0} & \equiv \int_{0}^{\infty} d t B_{22}(t) \\
\gamma_{c} & \equiv \int_{0}^{\infty} d t B_{22}(t) \cos (2 \Delta t) \\
\gamma_{s} & \equiv \int_{0}^{\infty} d t B_{22}(t) \sin (2 \Delta t)
\end{aligned}
$$

The relaxation part of Eq. (40) then reads 


$$
\begin{aligned}
\sum_{\alpha=1,2}\left\{\left[\Lambda_{\alpha} \rho, K_{\alpha}\right]+\text { h.c. }\right\}= & \frac{\gamma_{0}^{\prime}}{6}\left(\begin{array}{cc}
\rho_{22}-\rho_{11} & \rho_{12}^{*}-\rho_{12} \\
\rho_{12}-\rho_{12}^{*} & \rho_{11}-\rho_{22}
\end{array}\right)+\frac{\gamma_{c}^{\prime}}{6}\left(\begin{array}{cc}
\rho_{22}-\rho_{11} & -\rho_{12}^{*}-\rho_{12} \\
-\rho_{12}-\rho_{12}^{*} & \rho_{11}-\rho_{22}
\end{array}\right) \\
& +i \frac{\gamma_{s}^{\prime}}{6}\left(\begin{array}{cc}
\rho_{12}-\rho_{12}^{*} & 0 \\
0 & \rho_{12}^{*}-\rho_{12}
\end{array}\right)+\frac{\gamma_{s}^{\prime \prime}}{6}\left(\begin{array}{cc}
0 & 1 \\
1 & 0
\end{array}\right)
\end{aligned}
$$

where the single and double primes denote real and imaginary parts, respectively. They can be easily evaluated, yielding

$$
\begin{aligned}
\gamma_{0}^{\prime} & =0 \\
\gamma_{c}^{\prime}=\gamma_{s}^{\prime \prime} & =\frac{\pi}{2} \nu(2 \Delta) \operatorname{coth}\left(\frac{\Delta}{T}\right), \\
\gamma_{s}^{\prime} & =-f_{0}^{\infty} \frac{d y}{y^{2}-1} \nu(2 \Delta y) \operatorname{coth}\left(\frac{\Delta y}{T}\right) .
\end{aligned}
$$

The Liouville term in Eq. (40) is obtained straightforwardly:

$$
-i\left[\tilde{H}_{S}, \rho\right]=-i \Delta\left[\sigma_{1}, \rho\right]=-i \Delta\left(\begin{array}{cc}
\rho_{12}^{*}-\rho_{12} & \rho_{22}-\rho_{11} \\
\rho_{11}-\rho_{22} & \rho_{12}-\rho_{12}^{*}
\end{array}\right) .
$$

Introducing Eqs. (56) and (60) into (40), we obtain

$$
\begin{aligned}
& \dot{\rho}_{11}=-2\left(\Delta+\frac{\gamma_{s}^{\prime}}{6}\right) \rho_{12}^{\prime \prime}+\frac{\gamma_{c}^{\prime}}{6}\left(1-2 \rho_{11}\right), \\
& \dot{\rho}_{12}^{\prime}=-\frac{\gamma_{c}^{\prime}}{3} \rho_{12}^{\prime}+\frac{\gamma_{c}^{\prime}}{6}, \\
& \dot{\rho}_{12}^{\prime \prime}=-\Delta\left(1-2 \rho_{11}\right),
\end{aligned}
$$

where we have split the off-diagonal term $\rho_{12}$ into real and imaginary parts, $\rho_{12}^{\prime}+i \rho_{12}^{\prime \prime}$.

In order to identify energy and phase relaxation rates, we rewrite the elements of the reduced matrix in the eigenbasis of the system Hamiltonian,

$$
|E= \pm \Delta\rangle=\frac{1}{\sqrt{2}}(|+\rangle \pm|-\rangle)
$$

resulting in

$$
\begin{aligned}
& \dot{\tilde{\rho}}_{11}=-\frac{\gamma_{c}^{\prime}}{3} \tilde{\rho}_{11}+\frac{\gamma_{c}^{\prime}}{3}, \\
& \dot{\tilde{\rho}}_{12}^{\prime}=-\left(2 \Delta+\frac{\gamma_{s}^{\prime}}{3}\right) \tilde{\rho}_{12}^{\prime \prime}-\frac{\gamma_{c}^{\prime}}{3} \tilde{\rho}_{12}^{\prime}, \\
& \dot{\tilde{\rho}}_{12}^{\prime \prime}=2 b \tilde{\rho}_{12}^{\prime} .
\end{aligned}
$$

The solution of the diagonal term is straightforward,

$$
\tilde{\rho}_{11}^{\prime}(t)=1+\left[\tilde{\rho}_{11}^{\prime}(0)-1\right] e^{-\gamma_{c}^{\prime} t / 3},
$$

which allows us to read directly the energy relaxation time,

$$
T_{1}=\frac{3}{\gamma_{c}^{\prime}}
$$

For the off-diagonal term, one finds that the real part is given by

$$
\tilde{\rho}_{12}^{\prime}(t)=\tilde{\rho}_{12}^{\prime}(0) e^{-t / T_{2}} \cos \left(\omega_{c} t\right)
$$

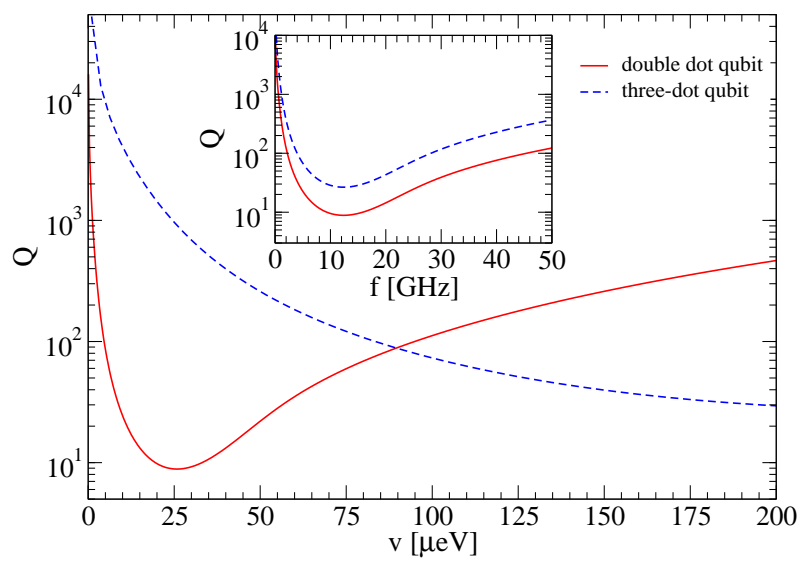

FIG. 4: (Color online) Comparison between the $Q$ factors of a threedot and a double-dot charge qubit coupled to piezoelectric acoustic phonons. The parameters used are: $a=60 \mathrm{~nm}, D=180 \mathrm{~nm}$, $s=5 \times 10^{3} \mathrm{~m} / \mathrm{s}, T=15 \mathrm{mK}$, and $g_{\mathrm{ph}}=0.05$, which correspond to realistic lateral quantum dot systems in GaAs. Here the variable $v$ denotes the interdot tunnel amplitude. Note that for double dot qubits, $\Delta=v$, while for three-dot qubits we assumed $\Delta=0.1 v$. In the inset we show the same $Q$ factors when the oscillation frequency (rather than $v$ ) is fixed. In this case the curves only differ by a factor of 3 .

where the phase relaxation time is equal to

$$
T_{2}=\frac{6}{\gamma_{c}^{\prime}}
$$

and the frequency of quantum oscillations is given by

$$
\omega_{c}=\sqrt{2 \Delta\left(2 \Delta+\frac{\gamma_{s}^{\prime}}{3}\right)-\frac{\gamma_{c}^{\prime 2}}{36}}
$$

Note that $T_{2}=2 T_{1}$, as well-known for the super-ohmic spinboson model in the weak-coupling regime. 38,39

Except for a factor of three in the relaxation rates, Eqs. (69)-(72) are identical to those found in Ref. 15 for a doubledot charge qubit. However, one has to recall that while in the double-dot qubit $\Delta$ is the interdot hopping matrix element $v$, for the three-dot qubit it takes a much smaller value, of the order of $\delta_{1,2,3}$. The decoherence times will be longer for the three-dot qubit, but so will be the quantum oscillation period and the single-qubit gate pulses. Therefore, it is meaningful to compare the quality factor of the the three-dot qubit to that obtained for the double-dot qubit for a fixed magnitude of $v$, which is a common experimental parameter to both setups. The comparison for the case of piezoelectric acoustic 
phonons and realistic GaAs quantum dot geometries (data for the double-dot qubit was obtained from Ref. 15) is shown in Fig. 4 The $Q$ factor is defined as

$$
Q=\frac{\omega_{c} T_{2}}{2 \pi} .
$$

We assume $\omega_{c} \approx 2 \Delta$ since $\Delta \gg \gamma_{c}^{\prime}, \gamma_{s}^{\prime}$ in the weak-coupling regime.

The improvement in the $Q$ factor is substantial for small tunnel amplitudes. A similar result was previously found by Storcz et al. in Ref.40 when considering the phonon-induced decoherence in a system of two double-dot charge qubits with a small tunnel splitting ("slow tunneling"). There, the dominant quadrupolar contribution to the two-qubit decoherence yields a $\omega_{c}^{5}$ dependence for the $Q$ factor. In our case, the extra protection in the three-dot qubit compared to the slow tunneling double-dot system arises mainly because the oscillation frequency $\omega_{c}$ (i.e., the amplitude of the transverse pseudomagnetic field) is smaller in the three-dot qubit by the ratio $\Delta / v$ [see Eq. (2)]. This ratio must be kept small in order to avoid leakage from the computational basis. In Fig. 4 it was set to 0.1 . However, for a fixed oscillation frequency (see inset in Fig. (4), the $Q$ factors for these two qubits differ by only an overall factor of three.

To summarize up to this point, our study indicates that using a computational basis with a homogeneous charge distribution improves the quality of the qubit but does not rid it from decoherence completely. The reason lies in the fact that bosonic modes propagating in the $x y$ plane can pick up distinct phase shifts when interacting with different dots [see Eqs. 37) to [39]. However, there is no complete destructive interference along any direction of propagation in the plane, as can be seen from Eqs. (35) and (36). In fact, one can show that the same is true for any ringlike array of dots that share a single excess electron.

\section{CHARGE QUBITS IN MULTIPOLE CONFIGURATIONS}

As recently proposed by Oi et al., 22 there is another way in which the geometry of the quantum dot qubit array and its charge distribution can be chosen to minimize the coupling to environmental degrees of freedom. Here we demonstrate how their idea can be extended to multiple-dot charge qubits coupled to gapless bosonic modes. It turns out that by reducing the computational space to particular multipole charge configurations one can substantially reduce the coupling to bath modes at low frequencies. We consider qubits and basis states as shown in Fig. 5] The qubit consists of a planar array of dots with alternating excess charge. Note that the operation of such a qubit is straightforward: The excess charge is only allowed to hop between every other pair of neighboring dots, namely, between dots numbered $2 n-1$ and $2 n$, with $n=1, \ldots, 2^{p-1}$, where $p$ is the multipole order, $l=2^{p}$ (see Fig. 5). Tunnel barriers between alternating pairs of dots must be maintained small and fixed (to avoid leakage), while the remaining barriers have to be modulated in time to implement an $X$ gate.
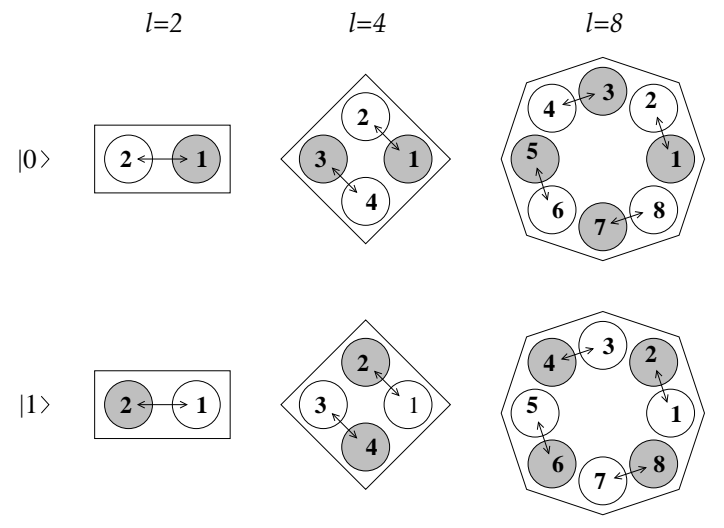

FIG. 5: The three lowest multipole charge qubit configurations (dipole, quadrupole, and octopole). The two computational basis states, $|0\rangle$ and $|1\rangle$, are indicated for for each configuration. Empty (filled) circles correspond to empty (occupied) quantum dots. The arrows indicate the pairs of quantum dots where excess charge can hop.

The $Z$ gate is implemented by inducing a small bias between even- and odd-numbered dots. Two-qubit operations can be implemented in analogy to the procedure discussed in Ref. 36 in the context of Ising-interaction based two-qubit operations.

The basis states for each multipole configuration have complementary charge distributions that tend to cancel out the coupling to phonon modes propagating along certain directions in the $x y$ plane. The number of such directions increases with the multipole order, resulting in an attenuation of the overall coupling to phonons at low frequencies (large wavelengths). The crossover frequency where this attenuation occurs is $\omega_{\text {cross }}^{(l)} \sim s / d_{l}$, where $d_{l}$ is the radius of the dot array. At high frequencies, however, when the phonon wavelength is much smaller than the radius $d_{l}$, decoherence becomes stronger because phonons can resolve the internal structure of the qubit and disturb charge motion between individual pairs of dots.

In order to demonstrate these effects, let us derive an expression for the spectral function of the qubit-bath system. For simplicity, we assume that all dots in the qubit are identical. In this case, the bath modes couple to charge variations in the dots according to the Hamiltonian

$$
H_{\mathrm{SB}}=\sum_{\mathbf{q}} \sum_{k=1}^{l} \alpha_{k} N_{k}\left(b_{\mathbf{q}}^{\dagger}+b_{-\mathbf{q}}\right),
$$

where $l=2^{p}$ and $N_{k}$ is the excess charge in the $k$ th dot. For the case of acoustic phonons, the coefficients $\alpha_{k}$ were defined in Eq. (30). Projecting this Hamiltonian onto the computational basis (as shown in Fig. 5), we find that, up to a constant term,

$$
H_{\mathrm{SB}}=K \Phi_{l},
$$

where $K=-\sigma_{z} / 2$ acts on the qubit space and

$$
\Phi_{l}=\sum_{\mathbf{q}} g_{\mathbf{q}}^{(l)}\left(b_{\mathbf{q}}^{\dagger}+b_{-\mathbf{q}}\right),
$$


acts on the phonon bath, with

$$
g_{\mathbf{q}}^{(l)}=\sum_{k=1}^{l}(-1)^{k} \alpha_{k}=\lambda_{\mathbf{q}} P_{\mathbf{q}} \sum_{k=1}^{l}(-1)^{k} e^{i \mathbf{R}_{k} \cdot \mathbf{q}} .
$$

It is convenient to choose the position vectors of the dots as $\mathbf{R}_{k}=d_{l}\left(\hat{x} \cos \varphi_{k}+\hat{y} \sin \varphi_{k}\right)$, where $\varphi_{k}=(2 \pi / l)(k-1)$ and $d_{l}$ is the array radius: $d_{l}=D / 2 \sin (\pi / l)$, where $D$ is the distance between neighboring dots. This yields

$$
\begin{aligned}
& \left|g_{\mathbf{q}}^{(l)}\right|^{2}=\left|\lambda_{\mathbf{q}} P_{\mathbf{q}}\right|^{2} \sum_{k, j=1}^{l}(-1)^{k+j} \\
& \quad \times \exp \left[2 i d_{l} q \sin \theta \sin \left(\varphi-\frac{\varphi_{k}+\varphi_{j}}{2}\right) \sin \left(\frac{\varphi_{k}-\varphi_{j}}{2}\right)\right],
\end{aligned}
$$

where $(q, \theta, \varphi)$ are the spherical coordinates of the wave vector q. It is not difficult to see that $g_{\mathbf{q}}=0$ for $\theta=\pi / 2$ and $\varphi=(2 m-1) \pi / l$, with $m=1, \ldots, l$.

The spectral function can now be obtained in analogy to the calculation shown in Sec. IID For a thermal bath of acoustic piezoelectric phonons, we find

$$
\begin{aligned}
\nu_{l}(\omega)= & \sum_{\mathbf{q}}\left|g_{\mathbf{q}}^{(l)}\right|^{2} \delta\left(\omega-\omega_{\mathbf{q}}\right) \\
= & \frac{g_{\mathrm{ph}} \omega l}{2} \int_{0}^{\pi / 2} d \theta \sin \theta \exp \left(-\frac{a^{2} \omega^{2} \sin ^{2} \theta}{s^{2}}\right) \\
& \times\left\{1+(-1)^{l / 2} J_{0}\left(\frac{2 d_{l} \omega}{s} \sin \theta\right)\right. \\
& \left.+2 \sum_{m=1}^{l / 2-1}(-1)^{m} J_{0}\left[\frac{2 d_{l} \omega}{s} \sin \theta \sin \left(\frac{m \pi}{l}\right)\right]\right\} .
\end{aligned}
$$

Implicit in Eq. (79) are the assumptions of in-plane isotropy of $\lambda_{\mathbf{q}}, P_{\mathbf{q}}$, and $\omega_{\mathbf{q}}$. Note that for $l=2$ one recovers the spectral function for a double dot qubit obtained in Ref.15. The lowfrequency behavior of the spectral density becomes apparent when we expand the Bessel functions in a power series, resulting in

$$
\begin{aligned}
\nu_{l}(\omega)= & \frac{g_{\mathrm{ph}} \omega l}{2} \int_{0}^{\pi / 2} d \theta \sin \theta \exp \left(-\frac{\omega^{2} a^{2}}{s^{2}} \sin ^{2} \theta\right) \\
& \times \sum_{k=1}^{\infty} \frac{(-1)^{k}}{(k !)^{2}}\left(\frac{d_{l} \omega}{s} \sin \theta\right)^{2 k} a_{k}^{(l)}
\end{aligned}
$$

where

$$
a_{k}^{(l)}=(-1)^{l / 2}+2 \sum_{m=1}^{l / 2-1}(-1)^{m} \sin ^{2 k}\left(\frac{m \pi}{l}\right) .
$$

It is possible to show that $a_{k}^{(l)}=0$ for $k<l / 2$ when $l$ is an integer power of 2 . Therefore, $\nu_{l}(\omega \rightarrow 0) \sim \omega^{l+1}$. For large $l$, this amounts to the appearance of a pseudo gap in the spectral function at low frequencies. The asymptotic behavior of the spectral function at high frequencies is also straightforward to obtain: One finds $\nu_{l}(\omega \rightarrow \infty) \sim l / \omega$. Thus, the tail of the spectral function raises with increasing multipole order.

The structure of the computational basis is simple enough to allow for the qubit to couple to just one bath mode (in contrast to the three-dot qubit, where two modes couple to the qubit). Thus, the standard expressions for the relaxation times in the spin-boson model can be used, ${ }^{39}$ The result is

$$
\gamma^{(l)}=\frac{\pi}{2} \nu_{l}(2 v) \operatorname{coth}\left(\frac{v}{T}\right)
$$

where $v$ is the inter-dot tunnel amplitude. Note that Eq. (82) reduces to the result found in Ref. 15 when $l=2$, as expected. Provided that $v$ is sufficiently smaller than the temperature, $\gamma^{(l)} \sim v^{l}$. Thus, by increasing the order of the multipole and maintaining a low frequency of operation, one can decrease the qubit relaxation rate by orders of magnitude without affecting the frequency of quantum oscillations. In Fig. 6 we show the $Q$ factor of several multipole charge qubits as a function of the inter-dot coupling $v$. Note that at low frequencies high quantum oscillations are much less damped for high multipole configurations. This translates into single-qubit gates of much higher fidelity. Clearly, this gain in the $Q$ factor has to be contrasted with the high complexity of operating a logical qubit comprised by a large number of quantum dots, as well with the slowness in operation. As the gating becomes slower, other sources of decoherence may become more relevant.

It is also important to remark that, in practice, the pseudogap width, $\omega_{\text {cross }}^{(l)}$, will shrink with increasing multipole order. This is because the dot array radius scales as $d_{l} \approx l D / 2 \pi$ for $l \gg 1$. Therefore, for a fixed value of $D$, one has $\omega_{\text {cross }}^{(l)} \sim$ $2 \pi s /(l D)$ for $l \gg 1$. Finally, we note that the results discussed above are valid for any gapless bosonic bath. Different dependences on $q$ for the coupling constant $\lambda_{q}$ and dispersion relation $\omega_{q}$ will only change the power of the frequencydependent prefactor in Eq. (80).

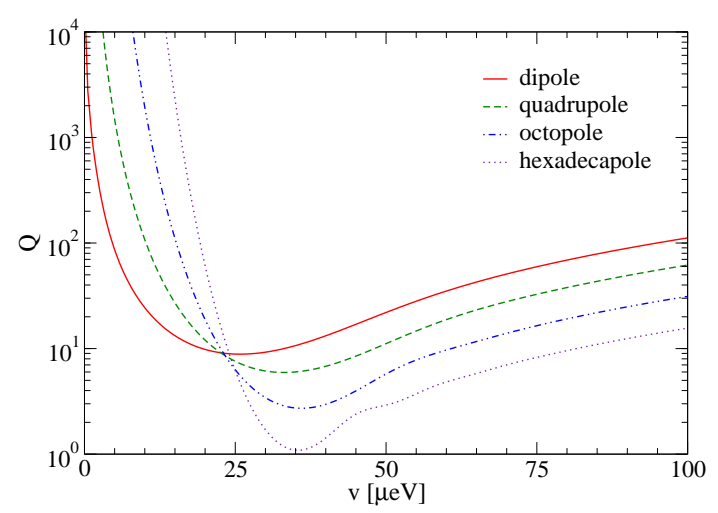

FIG. 6: (Color online) $Q$ factors for multipole charge qubits $(l=2,4,8,16)$ coupled to piezoelectric acoustic phonons: $Q_{l}=$ $\omega_{c} / \pi \gamma^{(l)}$, where $\omega_{c} \approx 2 v$ [for $\gamma^{(l)}$, see Eq. 82)]. Physical and geometrical parameters are the same as those used in Fig. 4 In particular, note that the inter-dot distance is fixed, $D=120 \mathrm{~nm}$, for all configurations. 


\section{CONCLUSIONS}

In this paper we have shown that, whereas there are no simple ways to completely protect charge qubits based on quantum dots from decoherence by gapless bosonic modes, a homogeneous charge distribution throughout the qubit is the most advantageous setup and provides the best possible protection against decoherence. This result applies not only to the charge qubits in semiconductor-heterostructures that we focused on here, but, in principle, to charge qubits in general. Whereas certain aspects of the discussion need to be changed for, say, self-assembled quantum dots, single-donor charge qubits, ${ }^{41}$ or Si-based quantum dot structures,, 20 this does not affect the universal mechanism underlying our central result, namely that a specific (homogeneous) charge distribution within the qubit enables the cancellation of (certain) decoherence modes.

Contrary to spin-based quantum dot qubits, where decoherence-free subspaces can be created by combining quantum dots into logical units, charge-based qubits are much more difficult to isolate from the environment. In order to have decoherence-free subspaces for charge qubits one would need to restrict the operation to a subspace where charge is homogeneously distributed in space, no matter which basis states are chosen. However, this contradicts the very nature of a charge qubit (where readout depends on charge imbalance) and thus cannot be achieved. In our example of the three-dot qubit these facts become evident in the existence of two phonon modes that cannot cancel due to geometric constraints inherent to the qubit.

Decoherence can be mitigated in a number of other ways. For instance, for the three-dot qubit case we have studied, a substantial improvement with respect to the double-dot qubit can be achieved due to the lower frequency of operation and to an enhancement of the relaxation time by a factor of three.

Another effective way to reduce the coupling to gapless bosonic modes is to choose a computational basis with a multipole charge configuration. As we have shown, the multipole geometry attenuates the coupling to long wavelength acoustic phonons by a factor proportional to a power law of the operation frequency. This power law grows rapidly with the multipole order. Thus, multipole configurations of charge can lead to quality factors enhanced by orders of magnitude in comparison to those obtained for double-dot qubits. However, the effect is reversed at high frequencies of operation.
The crossover frequency separating the two regimes is given by the inverse traversal time for a phonon to propagate across the qubit. For typical GaAs setups, this time is of the order of $30 \mathrm{ps}$ (for dots $120 \mathrm{~nm}$ apart), indicating a crossover frequency in the range of $30 \mathrm{GHz}$. Since tunnel amplitudes usually vary from tens to a few hundreds of $\mu \mathrm{eV}$, yielding quantum oscillations of about $2-20 \mathrm{GHz}$, there is a real advantage in moving toward multiple-dot qubits for current setups. However, since phonons are not the only leading mechanism for decoherence in charge qubits, 15 as operation frequencies go down other sources of decoherence, not necessarily modeled by bosonic environments, may become dominant. In that case multiple-dot qubits might become less appealing. Investigation of other decoherence mechanisms in multiple-dot qubits, such as electromagnetic environmental fluctuations and telegraph noise due to charges trapped in the substrate, are the subject of ongoing investigations.

Finally, we mention that a recent work has shown that pulse optimization is also a very effective way of minimizing the coupling to bosonic environments in quantum dot charge qubits. ${ }^{42}$

\section{ACKNOWLEDGMENTS}

We would like to thank J. Siewert for useful discussions. M.H. thanks the Alexander von Humboldt Foundation for partial support and also acknowledges funding through the Emmy-Noether program of the German Research Foundation (DFG). This work was also supported in part by the NSA and ARDA under ARO Contract No. DAAD19-02-1-0079 and by NSF Grants No. CCF-0523509 and No. CCF-0523603. D.C.B.V. and E.R.M. acknowledge partial support from the Interdisciplinary Information Science and Technology Laboratory $\left(\mathrm{I}^{2} \mathrm{Lab}\right)$ at $\mathrm{UCF}$.

\section{APPENDIX A: THE TWO-QUBIT REDUCED HAMILTONIAN}

The Hamiltonian of two three-dot qubits coupled by their bases [see Fig. 3 (b)] with inter-qubit couplings $t^{\prime}$ and $t^{\prime \prime}$ has the following matrix form in the basis of Eqs. (11)-(20) (the lower off-diagonal block is omitted): 


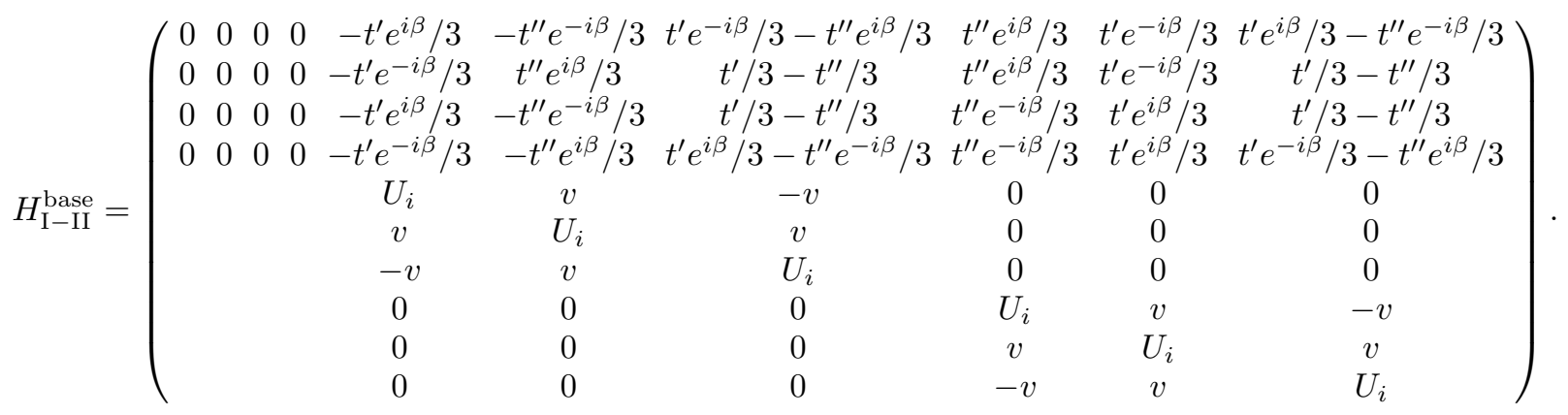

[Note that the Hamiltonian for the tip-tip configuration is recovered by setting $t^{\prime \prime}=0$ in Eq. A1).] This Hamiltonian can be projected onto the two-qubit computational subspace by means of a Schrieffer-Wolff transformation. From Eq. [A1, we see that the Hamiltonian has the form $H_{\mathrm{I}-\mathrm{II}}^{\text {base }}=H_{0}+H_{1}$, where

$$
H_{0}=\left(\begin{array}{cc}
0 & 0 \\
0 & M
\end{array}\right), \quad H_{1}=\left(\begin{array}{cc}
0 & T \\
T^{\dagger} & 0
\end{array}\right),
$$

and $M$ and $T$ are $6 \times 6$ and $4 \times 6$ matrices, respectively. Performing the Schrieffer-Wolff transformation and expanding to second order in $H_{1}$, 31 we get $\tilde{H}_{\mathrm{I}-\mathrm{II}}^{\text {base }} \approx H_{0}+(1 / 2)\left[S, H_{1}\right]$, where

$$
S=\left(\begin{array}{cc}
0 & -T M^{-1} \\
-M^{-1} T^{\dagger} & 0
\end{array}\right)
$$

Thus the Hamiltonian has the block diagonal structure

$$
\tilde{H}_{\mathrm{I}-\mathrm{II}}^{\mathrm{base}} \approx\left(\begin{array}{cc}
H_{\mathrm{I}}^{\mathrm{red}} & 0 \\
0 & M
\end{array}\right)
$$

where $H_{\mathrm{I}-I \mathrm{II}}^{\mathrm{red}}=-T M^{-1} T^{\dagger}$. The matrix $M$ can be broken into two identical $3 \times 3$ diagonal blocks,

$$
M=\left(\begin{array}{cc}
B & 0 \\
0 & B
\end{array}\right)
$$

and $T$ can be broken into two distinct $4 \times 3$ blocks,

$$
T=\left(\begin{array}{ll}
T_{\mathrm{I}} & T_{\mathrm{II}}
\end{array}\right) .
$$

As a result, $H_{\mathrm{I}-\mathrm{II}}^{\mathrm{red}}=-T_{\mathrm{I}} B^{-1} T_{\mathrm{I}}^{\dagger}-T_{\mathrm{II}} B^{-1} T_{\mathrm{II}}^{\dagger}$. After some algebra, one finds that

$$
B^{-1}=\frac{1}{3}\left(\begin{array}{ccc}
2 u_{1}+u_{2} & u_{1}-u_{2} & -u_{1}+u_{2} \\
u_{1}-u_{2} & 2 u_{1}+u_{2} & u_{1}-u_{2} \\
-u_{1}+u_{2} & u_{1}-u_{2} & 2 u_{1}+u_{2}
\end{array}\right),
$$

where $u_{1}=\left(U_{i}+v\right)^{-1}$ and $u_{2}=\left(U_{i}-2 v\right)^{-1}$. The structure of $B^{-1}$ can be substantially simplified by assuming $v \ll U_{i}$ and neglecting $v$. This yields $H_{\mathrm{I}-\mathrm{II}}^{\mathrm{red}}=$ $\left(-1 / U_{i}\right)\left(T_{\mathrm{I}} T_{\mathrm{I}}^{\dagger}+T_{\mathrm{II}} T_{\mathrm{II}}^{\dagger}\right)$. Carrying out the matrix multiplications and setting $t^{\prime \prime}=0$, we obtain Eq. (23).
${ }^{1}$ Semiconductor Spintronics and Quantum Computation, edited by D. D. Awschalom, D. Loss, and N. Samarth, (Springer, Berlin, 2002).

2 D. Loss and D. P. DiVincenzo, Phys. Rev. A 57, 120 (1998).

3 D. P. DiVincenco, D. Bacon, J. Kempe, G. Burkhard, and K. B. Whaley, Nature (London) 408, 339, (2000).

4 J.R. Petta, A. C. Johnson, J. M. Taylor, E. A. Laird, A. Yacoby, M. D. Lukin, C. M. Marcus, M. P. Hanson, A. C. Gossard, Science 309, 2180 (2005).

${ }^{5}$ F. H. L. Koppens, C. Buizert, K. J. Tielrooij, I. T. Vink, K. C. Nowack, T. Meunier, L. P. Kouwenhoven and L. M. K. Vandersypen, Nature (London) 442, 766 (2006).

6 J. Levy, Phys. Rev. Lett. 89, 147902 (2002); Y. S. Weinstein and C. S. Hellberg, Phys. Rev. A 72, 022319 (2005); Phys. Rev. Lett.
98, 110501 (2007).

7 Yu. A. Pashkin, T. Yamamoto, O. Astafiev, Y. Nakamura, D. V. Averin, and J. S. Tsai, Nature (London) 421, 823 (2003).

${ }^{8}$ D. Vion, A. Aassime, A. Cottet, P. Joyez, H. Pothier, C. Urbina, D. Estève, and M. H. Devoret, Science 296, 886 (2002).

9 A. Blais, J. Gambetta, A. Wallraff, D. I. Schuster, S. M. Girvin, M. H. Devoret, and R. J. Schoelkopf, Phys. Rev. A 75, 032329 (2007).

10 J. Koch, T. M. Yu, J. Gambetta, A. A. Houck, D. I. Schuster, J. Majer, A. Blais, M. H. Devoret, S. M. Girvin, R. J. Schoelkopf, Phys. Rev. A 76, 042319 (2007).

11 R. H. Blick and H. Lorenz, in Proceedings of the IEEE International Symposium on Circuits and Systems, edited by J. Calder (IEEE, Piscataway, NJ, 2000), Vol. II, p. 245. 
12 T. Tanamoto, Phys. Rev. A 61, 022305 (2000).

${ }^{13}$ L. Fedichkin, M. Yanchenko, and K. A. Valiev, Nanotechnology 11, 387 (2000); L. Fedichkin and A. Fedorov, Phys. Rev. A 69, 032311 (2004).

14 T. Brandes and T. Vorrath, Phys. Rev. B 66, 075341 (2002).

15 S. Vorojtsov, E. R. Mucciolo, and H. U. Baranger, Phys. Rev. B 71, 205322 (2005).

16 Z.-J. Wu, K.-D. Zhu, X.-Z. Yuan, Y.-W. Jiang, and H. Zheng, Phys. Rev. B 71, 205323 (2005).

17 V. N. Stavrou and X. Hu, Phys. Rev. B 72, 075362 (2005).

18 T. Hayashi, T. Fujisawa, H. D. Cheong, Y. H. Jeong, and Y. Hirayama, Phys. Rev. Lett. 91, 226804 (2003); T. Fujisawa, T. Hayashi, H. D. Cheong, Y. H. Jeong, and Y. Hirayama, Physica E 21, 1046 (2004).

19 J. R. Petta, A. C. Johnson, C. M. Marcus, M. P. Hanson, and A. C. Gossard, Phys. Rev. Lett. 93, 186802 (2004).

${ }^{20}$ J. Gorman, D. G. Hasko, and D. A. Williams, Phys. Rev. Lett. 95, 090502 (2005).

${ }^{21}$ J. Kempe, D. Bacon, D. A. Lidar, and K. B. Whaley, Phys. Rev. A 63, 042307 (2001).

${ }^{22}$ D. K. L. Oi, S. G. Schirmer, A. D. Greentree, and T. M. Stace, Phys. Rev. B 72, 075348 (2005).

${ }^{23}$ I. O. Kulik, T. Hakioğlu, and A. Barone, Eur. Phys. J. B 30, 219 (2002).

${ }^{24}$ M. Thorwart, J. Eckel, and E. R. Mucciolo, Phys. Rev. B 72, 235320 (2005).

25 P. N. Argyres and P. L. Kelley, Phys. Rev. 134, A98 (1964).

${ }^{26}$ The detailed electronic structure of model quantum dots in a triangular configuration has been studied in, for instance, M. Korkusinski, I. P. Gimenez, P. Hawrylak, L. Gaudreau, S. A. Studenikin, and A. S. Sachrajda, Phys. Rev. B 75, 115301 (2007) and F. Delgado, Y.-P. Shim, M. Korkusinski, and P. Hawrylak, Phys. Rev. B 76, 115332 (2007).

${ }^{27}$ By working with 1 hole per three-dot qubit (ie. 5 electrons in 3 levels) instead of 1 electron, the degeneracy between eigenstates $|+\rangle$ and $|-\rangle$ occurs at $B=0$, which may have some advantages.

28 J. E. Mooij, T. P. Orlando, L. Levitov, L. Tian, C. H. van der Wal, and S. Lloyd, Science 285, 1036 (1999).

29 L. Tian and P. Zoller, Phys. Rev. A 68, 042321 (2003).
30 A more technical argument for neglecting the states $|T\rangle_{\mathrm{I}}$ and $|T\rangle_{\mathrm{II}}$ can be constructed as follows. First, keep all such states while performing a first Schrieffer-Wolff transformation to find the effect of virtual excitation to the doubly-occupied states. This yields an effective Hamiltonian in the space of singly-occupied states with magnitude of order $t^{\prime 2} / U_{i}$. It includes off-diagonal terms between the computational basis and the $|T\rangle$ states. Now, perform a second Schrieffer-Wolff transformation relying on the fact that $t^{\prime 2} / U_{i} \ll v$ to integrate out the $|T\rangle$ states. The contribution of this second transformation to the effective Hamiltonian in the computational basis is of order $\left(t^{\prime 2} / U_{i}\right)^{2} / v$. As this is much smaller than $t^{\prime 2} / U_{i}$, the effect of the $|T\rangle$ states may be safely neglected.

31 J. R. Schrieffer and P. A. Wolff, Phys. Rev. 149, 491 (1966).

${ }^{32}$ N. Schuch and J. Siewert, Phys. Rev. A 67, 032301 (2003).

33 This Hamiltonian can be easily derived for the case of phonons, e.g., E. R. Mucciolo, S. Vorojtsov, and H. U. Baranger, in Quantum Information and Computation III, edited by E. J. Donkor, A. R. Pirich, and H. E. Brandt, Proceedings of the SPIE 5815, 53 (2005).

34 A. J. Leggett, S. Chakravarty, A. T. Dorsey, Matthew P. A. Fisher, Anupam Garg, and W. Zwerger, Rev. Mod. Phys. 59, 1 (1987).

35 W. T. Pollard and R. A. Friesner, J. Chem. Phys. 100, 5054 (1994).

${ }^{36}$ M. A. Nielsen and I. L. Chuang, Quantum Computation and Quantum Information (Cambridge University Press, Cambridge, U.K., 2000).

${ }^{37}$ H. Bruus, K. Flensberg, and H. Smith, Phys. Rev. B 48, 11144 (1993).

38 C. P. Slichter, Principles of Magnetic Resonance, 3rd ed. (Springer, Berlin, 1996).

39 U. Weiss, Quantum Dissipative Systems (World Scientific, Singapore, 1999), Sec. 21.5.2.

40 M. J. Storcz, U. Hartmann, S. Kohler, and F. K. Wilhelm, Phys. Rev. B 72, 235321 (2005).

${ }^{41}$ L. C. L. Hollenberg, A. S. Dzurak, C. Wellard, A. R. Hamilton, D. J. Reilly, G. J. Milburn, and R. G. Clark, Phys. Rev. B 69, 113301 (2004).

${ }^{42}$ U. Hohenester, Phys. Rev. B 74, 161307(R) (R) (2006). 